\title{
Pulmonary cysts identified on chest CT: are they part of aging change or of clinical significance?
}

\author{
Tetsuro Araki, ${ }^{1}$ Mizuki Nishino, ${ }^{1}$ Wei Gao, ${ }^{2}$ Josée Dupuis, ${ }^{2,3}$ Rachel K Putman, ${ }^{4}$ \\ George R Washko, ${ }^{4}$ Gary M Hunninghake, ${ }^{4}$ George T O'Connor, ${ }^{3,5}$ Hiroto Hatabu ${ }^{1}$
}

- Additional material is published online. To view please visit the journal (http:// dx.doi.org/10.1136/thoraxjnl2015-207653).

1 Department of Radiology, Center for Pulmonary Functional Imaging, Brigham and Women's Hospital, Harvard Medical School, Boston, Massachusetts, USA ${ }^{2}$ Department of Biostatistics, Boston University School of Public Health, Boston, Massachusetts, USA

${ }^{3}$ The National Heart Lung and Blood Institute's Framingham Heart Study, Framingham, Massachusetts, USA

${ }^{4}$ The Pulmonary and Critical Care Division, Brigham and Women's Hospital, Harvard Medical School, Boston, Massachusetts, USA

${ }^{5}$ Pulmonary Center and Department of Medicine, Boston University School of Medicine, Boston,

Massachusetts, USA

\section{Correspondence to} Dr Tetsuro Araki, Department of Radiology, Center for Pulmonary Functional Imaging, Brigham and Women's Hospital, Harvard Medical School, 75 Francis St., Boston, MA 02215, USA; taraki@partners.org

Received 31 July 2015 Revised 4 September 2015 Accepted 1 October 2015 Published Online First 29 October 2015

\section{ABSTRACT \\ Objective To investigate the prevalence and natural course of pulmonary cysts in a population-based cohort and to describe the CT image characteristics in association with participant demographics and pulmonary functions.}

Materials and methods Chest CT scans of 2633 participants (mean age 59.2 years; $50 \%$ female) of the Framingham Heart Study (FHS) were visually evaluated for the presence of pulmonary cysts and their image characteristics. These findings were correlated with participant demographics and results of pulmonary function tests as well as the presence of emphysema independently detected on CT. The interval change was investigated by comparison with previous CT scans (median interval 6.1 years).

Results Pulmonary cysts were seen in $7.6 \%(95 \% \mathrm{Cl}$ $6.6 \%$ to $8.7 \% ; 200 / 2633)$. They were not observed in participants younger than 40 years old, and the prevalence increased with age. Multiple cysts (at least five) were seen in $0.9 \%$ of all participants. Participants with pulmonary cysts showed significantly lower body mass index (BMI) $(p<0.001)$. Pulmonary cysts were most likely to appear solitary in the peripheral area of the lower lobes and remain unchanged or slightly increase in size over time. Pulmonary cysts showed no significant influence on pulmonary functions ( $p=0.07-0.6)$ except for diffusing capacity of the lung for carbon monoxide (DLCO) $(p=0.03)$ and no association with cigarette smoking $(p=0.1-0.9)$ or emphysema $(p=0.7)$.

Conclusions Pulmonary cysts identified on chest CT may be a part of the aging changes of the lungs, occurring in asymptomatic individuals older than 40 years, and are associated with decreased BMI and DLCO. Multiple pulmonary cysts may need to be evaluated for the possibility of cystic lung diseases.

\section{INTRODUCTION}

A pulmonary cyst is defined as a round, usually thinwalled, parenchymal lucency or low-attenuating area with a well defined interface with normal lung on chest $\mathrm{CT}^{1}{ }^{1}$ Incidental findings of pulmonary cysts are becoming more common because of the widespread use of CT scans in daily clinical practice and in lung cancer screening. Multiple pulmonary cysts are identified in various diseases such as pulmonary Langerhans cell histiocytosis (PLCH), lymphangioleiomyomatosis (LAM) and lymphoid interstitial pneumonia (LIP). ${ }^{2}$ These progressive diseases are often symptomatic and may result in impairment of pulmonary functions. In contrast, solitary or sporadic

\section{Key messages}

What is the key question?

- What is the significance of pulmonary cysts identified on chest $\mathrm{CT}$ ?

\section{What is the bottom line?}

- Solitary or sporadic pulmonary cysts are associated with the aging of the lungs and multiple cysts (at least five), which are observed on $\mathrm{CT}$ images in less than $1 \%$ of participants, may be scrutinised for the possibility of cystic lung diseases.

\section{Why read on?}

- This study provides an epidemiological overview of pulmonary cysts and reveals their imaging features, which may be useful for managing individuals with pulmonary cysts incidentally detected on CT.

pulmonary cysts can be incidentally seen on chest CT of otherwise healthy individuals. In a study by Copley et $a l,{ }^{3}$ pulmonary cysts were seen on CT in $25 \%(10 / 40)$ of individuals older than 75 years but in no individuals younger than 55 years $(0 / 16)$. More recently, Winter $e t a l^{4}$ also reported pulmonary cysts were seen in 13\% (6/47) of individuals older than 65 years but none in those aged $30-50$ years $(0 / 24)$. These studies suggest that asymptomatic pulmonary cysts could be a part of the aging processes. Other age-related findings of the lungs were reticular pattern, fibrotic changes, bronchial wall thickening and air trapping. ${ }^{3-8}$ It is important to differentiate asymptomatic pulmonary cysts from progressive cystic lung diseases or emphysema. However, there is no systematic study that has investigated the clinical impact of pulmonary cysts in a large cohort. There is a clear gap in knowledge in the prevalence and management of pulmonary cysts. We hypothesise that most incidentally found pulmonary cysts could be regarded as a part of the aging changes.

The purpose of the present study was to investigate the prevalence and natural course of pulmonary cysts in the Framingham Heart Study (FHS) cohorts and to describe their CT imaging characteristics in association with participant demographics and pulmonary functions, which may help clinically manage pulmonary cysts found on chest CT. 


\section{MATERIALS AND METHODS}

\section{Study population}

The original cohort of the FHS was recruited in $1948 .{ }^{9}$ Subsequently, the Offspring cohort, which consists of children of the original cohort members and their spouses, was recruited in 1971, followed by the Third Generation cohort in 2002, consisting of the grandchildren of the original cohort members. From 2009 to 2011, 2764 participants of the Offspring and the Third Generation cohorts underwent a non-contrast chest CT scan (FHS-MDCT2) in the supine position at full inspiration using a 64-detector-row scanner (Discovery, GE Healthcare, Waukesha, Wisconsin, USA) with $120 \mathrm{kV}, 300-350 \mathrm{~mA}$, gantry rotation time of $0.35 \mathrm{~s}$ and section thickness of $0.63 \mathrm{~mm}$. Of those, 131 were missing CT image data. Therefore, 2633 participants (mean age 59.2 years; SD 12.0; range 34-92 years; 50\% female) who had chest CT scans were included. From 2002 to 2005, many of those participants previously underwent an ECG-gated non-contrast cardiac CT scan (FHS-MDCT1) using an 8-detector-row scanner (Lightspeed, GE Healthcare, Waukesha, Wisconsin, USA) with $120 \mathrm{kV}, 320-400 \mathrm{~mA}$, a gantry rotation time of $0.5 \mathrm{~s}$ and section thickness of $2.5 \mathrm{~mm}$. The coverage of the scan is from $2 \mathrm{~cm}$ below the carina to the apex of the heart. The institutional review boards at both Brigham and Women's Hospital and Boston University approved the present study. Written informed consent was obtained from all participants upon the enrolment of the FHS.

\section{Evaluation of chest $\mathrm{CT}$ images}

Chest CT scans (MDCT2) of 2633 participants were evaluated on a workstation (Virtual Place Raijin, AZE Ltd, Tokyo, Japan) for pulmonary cysts with a fixed lung window setting (WL= -700 , WW $=1500)$. Pulmonary cysts are defined as a round, thin-walled parenchymal lucency or low-attenuating area with a well defined interface with normal lung ${ }^{1}$ (figure 1 ). The evaluation process was divided into two phases: detection of pulmonary cysts, and characterisation and measurement.

First, CT scans were reviewed for the presence of pulmonary cysts ( 0 : absent, 1 : present) by three board-certified radiologists (TA, HH, MN) using a modified sequential reading method as previously described. ${ }^{10}{ }^{11}$ Reader 1 reviewed all CT scans for screening of pulmonary cysts, providing a score of 0 or 1 for

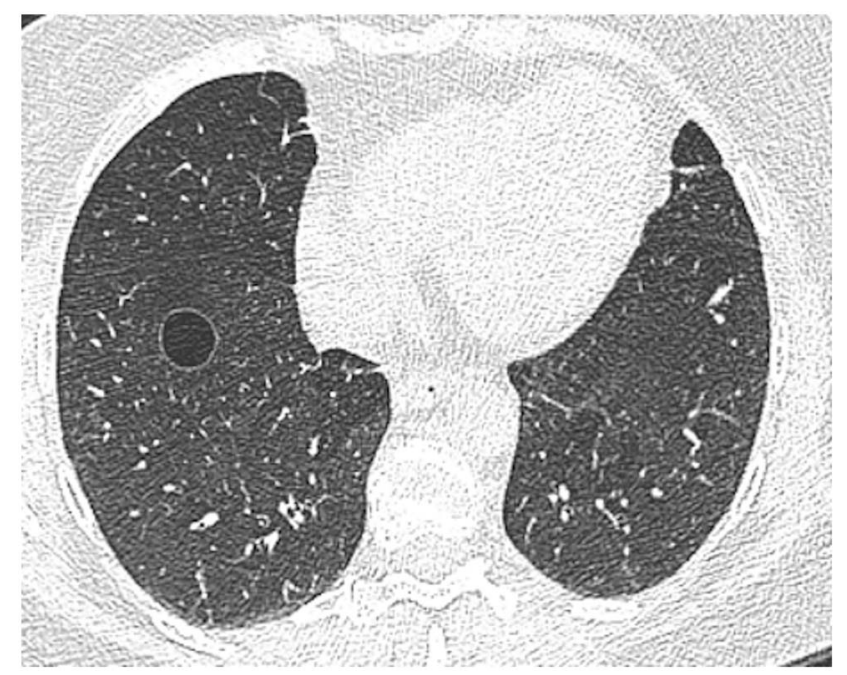

Figure 1 A transaxial CT image of a 60-year-old woman who is a former smoker with 4.2 pack-years shows a solitary cyst in the right lower lobe of the lung. The cyst is circumscribed with a thin wall and measures $17 \mathrm{~mm}$ in diameter. each case. Reader 1 repeated the review of cases scored 0 in the first review; two sets of scores were combined to increase the sensitivity. After the evaluations by Reader 1, cases with score 1 and approximately $20 \%$ of those with score 0 were forwarded to the next review by Reader 2. Finally, Reader 3 reviewed discordant cases between the first and second readers. Final scores were determined with the majority opinion of all three readers. $^{10} 11$

Second, cases identified with pulmonary cysts were reviewed by consensus of Readers 1 and 2 for the following image features: number of cysts (solitary $=1$, sporadic $=2-4$, multiple $\geq 5$ ); lobe of the lungs (upper, middle and lingular, or lower); location within the lobe (central, peripheral, subpleural); image findings of the cyst (calcification, penetration of blood vessels, fluid accumulation). The longest diameter of the pulmonary cyst was measured using a caliper-type measurement tool by Reader 1. Although most of the cases had a solitary cyst, if participants had more than one cyst in the lungs, the largest and most distinct cyst was regarded as representative of the case and evaluated for the imaging features and measurements. In addition, the presence of emphysema on chest CT scans was provided from a previous study (unpublished data). ${ }^{11}$ In brief, chest CT scans were evaluated in three lung sections (upper, middle, lower) for the presence of emphysema using the modified sequential reading method in the same manner as the evaluation for pulmonary cysts.

The demographics of participants, including age, sex, body mass index (BMI), smoking status with pack-years, and the results of pulmonary function tests $\left(\mathrm{FEV}_{1}, \mathrm{FVC}, \mathrm{DLCO}, \mathrm{TLC}\right)$ were obtained from the exams closest to the date of chest CT scan and were compared based on the result of CT image evaluations of pulmonary cysts.

Prior cardiac CT scans (MDCT1, median 6.1 years prior, range 4.0-8.4 years) were reviewed for comparison when available. Interval increase or decrease in size of the cyst was determined by changes in diameter of more than $2 \mathrm{~mm}$, accounting

Table 1 Image characteristics of pulmonary cysts

\begin{tabular}{|c|c|c|c|}
\hline & Frequency & $\begin{array}{l}\text { Per cent in } \\
\text { participants with } \\
\text { pulmonary cysts } \\
(\mathrm{N}=200)\end{array}$ & $\begin{array}{l}\text { Per cent in } \\
\text { all participants } \\
(\mathrm{N}=2633)\end{array}$ \\
\hline \multicolumn{4}{|l|}{ Number of cysts } \\
\hline Solitary (1) & 128 & 64 & 4.9 \\
\hline Sporadic (2-4) & 49 & 24.5 & 1.9 \\
\hline Multiple ( $\geq 5$ ) & 23 & 11.5 & 0.9 \\
\hline \multicolumn{4}{|l|}{ Lobe of the lungs } \\
\hline Lower & 131 & 65.5 & 5 \\
\hline Upper & 44 & 22 & 1.7 \\
\hline $\begin{array}{l}\text { Middle and } \\
\text { lingular }\end{array}$ & 25 & 12.5 & 1 \\
\hline \multicolumn{4}{|c|}{ Location in the lobe } \\
\hline Peripheral & 119 & 59.5 & 4.5 \\
\hline Subpleural & 62 & 31 & 2.4 \\
\hline Central & 19 & 9.5 & 0.7 \\
\hline \multicolumn{4}{|l|}{ Calcification } \\
\hline Absent & 199 & 99.5 & 7.6 \\
\hline Present & 1 & 0.5 & 0.04 \\
\hline \multicolumn{4}{|c|}{ Vascular penetration } \\
\hline Absent & 180 & 90 & 6.8 \\
\hline Present & 20 & 10 & 0.8 \\
\hline
\end{tabular}


Table 2 Participant demographics based on existence of pulmonary cysts

\begin{tabular}{|c|c|c|c|}
\hline & $\begin{array}{l}\text { No pulmonary cysts } \\
(\mathrm{N}=2433)\end{array}$ & $\begin{array}{l}\text { Pulmonary cysts } \\
(\mathrm{N}=200)\end{array}$ & $\begin{array}{l}p \\
\text { value }{ }^{*} \dagger\end{array}$ \\
\hline Age, years & $\begin{array}{l}58.9 \pm 12.0 \\
\text { (range 34.0-92.0) }\end{array}$ & $\begin{array}{l}63.0 \pm 12.0 \\
\text { (range } 40.1-89.8 \text { ) }\end{array}$ & $<0.001$ \\
\hline Female sex, n (\%) & $1229(51)$ & $96(49)$ & 0.5 \\
\hline Body mass index $\ddagger$ & $28.6 \pm 5.4(\mathrm{~N}=2420)$ & $27.1 \pm 4.7$ & $<0.001$ \\
\hline \multicolumn{4}{|l|}{ Smoking status, n (\%) } \\
\hline Never & $1188(49)$ & $80(40)$ & 0.1 \\
\hline Former & $1088(45)$ & $108(54)$ & 0.2 \\
\hline Current & $\begin{array}{l}150(6) \\
(N=2426)\end{array}$ & $12(6)$ & 0.7 \\
\hline Pack-years & $\begin{array}{l}17.9 \pm 18.1 \\
(\mathrm{~N}=1238)\end{array}$ & $\begin{array}{l}19.8 \pm 17.6 \\
(\mathrm{~N}=120)\end{array}$ & 0.9 \\
\hline $\begin{array}{l}\text { Coronary artery calcium } \\
\text { score§ }\end{array}$ & $\begin{array}{l}190.2 \pm 530.9 \\
(\mathrm{~N}=2303)\end{array}$ & $\begin{array}{l}167.9 \pm 344.7 \\
(N=188)\end{array}$ & 0.006 \\
\hline \multicolumn{4}{|c|}{ Respiratory symptoms, $\mathrm{n}(\%)$} \\
\hline Chronic cough & $168(7)$ & $8(4)$ & 0.8 \\
\hline $\begin{array}{l}\text { Shortness of breath } \\
\text { with minor exertion }\end{array}$ & $273(11)$ & $18(9)$ & 0.051 \\
\hline
\end{tabular}

Plus-minus values are mean \pm SD.

* $p$ values were calculated with the use of mixed effect models or generalised estimation equations to account for familial relationships in the Framingham Heart Study, as described previously. ${ }^{15}$

$t p$ values for body mass index, smoking status, pack-years, coronary artery calcium score and respiratory symptoms were adjusted for age and sex. In addition, the result for coronary artery calcium score was adjusted for the presence of emphysema; the results for respiratory symptoms were adjusted for smoking status and pack-years. ¥The body mass index is the weight in kilograms divided by the square of the height in metres.

$\S$ Coronary artery calcium scores were calculated by the Agatston method. ${ }^{27}$

for a possible variability based on the results of the preliminary study described as follows. ${ }^{12}$ Two radiologists (TA, HH) performed measurements of the cysts in 50 cases randomly selected from those with pulmonary cysts detected in the first phase of the evaluation. The first reader made the measurement twice separated by more than 6 months and the second reader did once.

\section{Statistical analysis}

Interobserver agreement of visual evaluation of pulmonary cysts was indicated with $\kappa$ values calculated using MedCalc (V.14.8.1, MedCalc Software, Ostend, Belgium). ${ }^{13}$ Intraobserver and interobserver measurement variability in the diameter of pulmonary cysts were evaluated with concordance correlation coefficient (CCC) and Bland-Altman plots with mean and 95\% limits of absolute difference of diameter, calculated or created also using MedCalc. ${ }^{14}$

All the other statistical analyses were performed using $\mathrm{R}$ (V.3.1.1, The R Foundation for Statistical Computing, Vienna, Austria). Participant demographics were investigated using mixed effect models for quantitative variables (age, BMI, packyears and coronary artery calcium scores) and generalised estimating equations for categorical variables (sex, smoking status and respiratory symptoms) to account for familial correlations in the cohorts. ${ }^{15}$ All the results for the demographics, including BMI, smoking status, pack-years, coronary artery calcium scores and respiratory symptoms were adjusted for age and sex. In addition, the result for coronary artery calcium scores was adjusted for the presence of emphysema; the results for respiratory symptoms were adjusted for smoking status and pack-years. The results of pulmonary function tests were compared between those with and without pulmonary cysts with adjustments for age, smoking status and pack-years. The associations between the presence of pulmonary cysts and emphysema, and between the image finding of vascular penetration and emphysema were evaluated with the Pearson $\chi^{2}$ test. $p$ values were from two-sided tests and regarded as statistically significant at the level of 0.05 .

\section{RESULTS}

Of 2633 participants, $200(7.6 \%$; 95\% CI 6.6\% to 8.7\%) were identified with at least one pulmonary cyst on chest CT scans. Interobserver agreement regarding the presence of pulmonary cysts between the first and second readers was substantial $(\kappa=0.75 ; 95 \%$ CI 0.70 to 0.81$)$. In the cases with score 0 provided by the first reader and subsequently reviewed by the second reader, the agreement rate between the first and second readers was $98.4 \%$. Image characteristics of pulmonary cysts are summarised in table 1 . Sixty-four per cent of pulmonary cysts $(128 / 200)$ were solitary, $65.5 \%(131 / 200)$ were in the lower lobe of the lungs and $59.5 \%(119 / 200)$ were located in the peripheral area. Vascular penetration was seen in 10\% (20/200); of these, only four cases were accompanied by emphysema. There was no significant association between the specific finding of vascular penetration and emphysema $(p=0.61)$. Calcification

Table 3 Characteristics of participants with pulmonary cyst stratified with smoking status

\begin{tabular}{|c|c|c|c|}
\hline & Non-smoker $(\mathrm{N}=80)$ & Former and current smoker $(\mathrm{N}=120)$ & p value* \\
\hline Age, years & $61.9 \pm 12.6$ (range $40.1-89.8$ ) & $63.7 \pm 61.9$ (range $41-88.9$ ) & 0.3 \\
\hline Female sex, $\mathrm{n}(\%)$ & $37(46)$ & $59(49)$ & 0.7 \\
\hline Body mass indext & $26.8 \pm 4.2$ & $27.3 \pm 5.0$ & 0.5 \\
\hline Emphysema, n (\%) & $1(1)$ & $24(20)$ & 0.002 \\
\hline Cyst size (diameter), mm & $11.2 \pm 6.1$ (range $4.0-34.3$ ) & $10.3 \pm 4.5$ (range $4.0-30.3$ ) & 0.2 \\
\hline \multicolumn{4}{|l|}{ Interval change in diameter, $\mathrm{n}(\%)$} \\
\hline Increased & $15(38)$ & $24(35)$ & 0.7 \\
\hline Unchanged & $23(58)$ & $45(65)$ & 0.4 \\
\hline Decreased & $\begin{array}{l}2(5) \\
(N=40)\end{array}$ & $\begin{array}{l}0(0) \\
(N=69)\end{array}$ & - \\
\hline Coronary artery calcium score $\ddagger$ & $158.1 \pm 331.1(\mathrm{~N}=77)$ & $174.7 \pm 355.1(\mathrm{~N}=111)$ & 0.98 \\
\hline
\end{tabular}


Figure $2 \mathrm{CT}$ images of a 47-year-old woman show a solitary round cyst (arrow) with a thin wall in the middle lobe of the right lung (A). She is a former smoker with 18 pack-years. Low attenuation colour-mapping overlaid images (threshold of at transaxial (B) and sagittal reconstruction $(C)$ demonstrate the distinct solitary pulmonary cyst (arrows) with the background lung parenchyma with a diffuse infiltration of mild emphysema. $-950 \mathrm{HU}$, indicated in light blue areas)

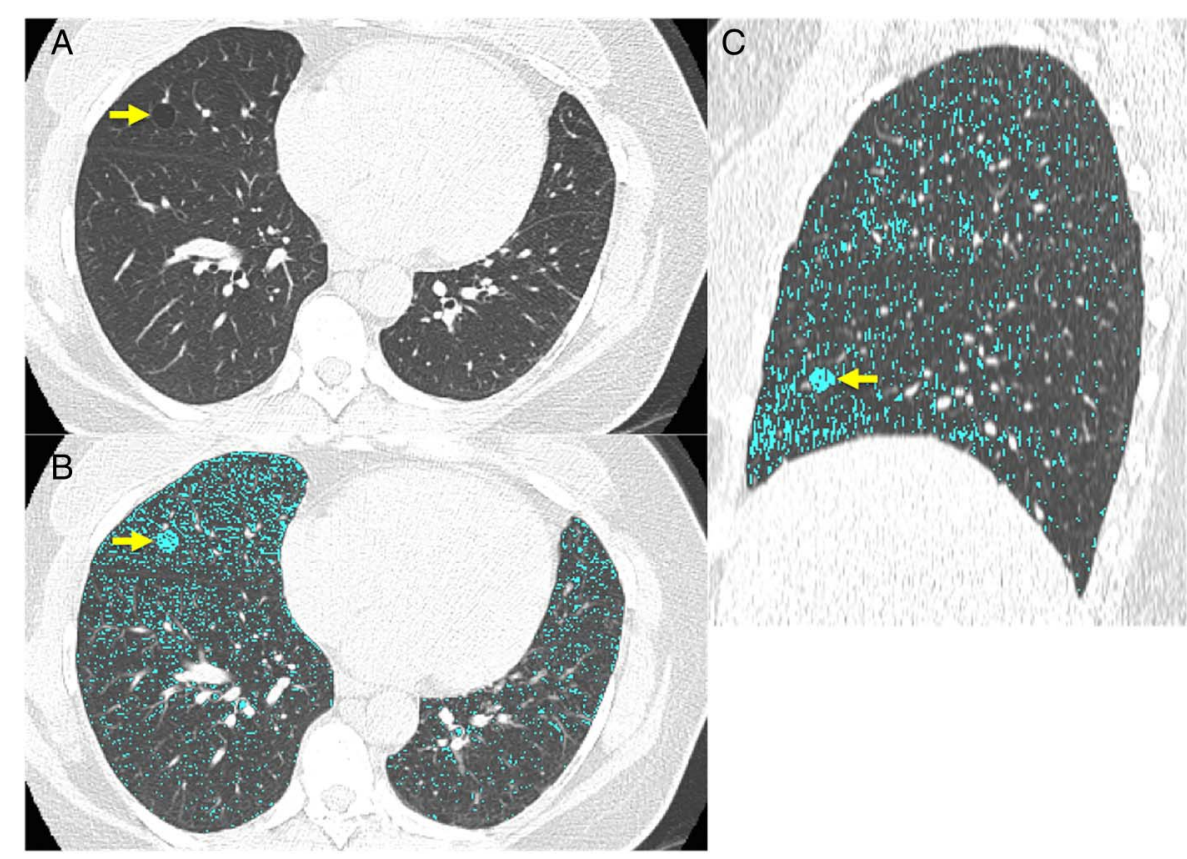

was seen in only one case $(0.5 \%)$. No pulmonary cysts appeared with a fluid collection.

Participant demographics in association with pulmonary cysts are summarised in table 2. Mean age of participants with pulmonary cysts was 63.0 years, significantly higher than those without pulmonary cysts $(58.9$ years, $\mathrm{p}<0.001)$. BMI of participants with pulmonary cysts (27.1) was significantly lower than those without pulmonary cysts $(28.6, \mathrm{p}<0.001)$. There were no significant differences in smoking status $(p=0.1-0.7)$ and packyears $(p=0.9)$. Means of coronary artery calcium scores were significantly different between the two groups $(p=0.006)$, but the score tended to be lower in participants with pulmonary cysts than in those without pulmonary cysts. There was a trend towards fewer reports of respiratory symptoms in those with pulmonary cysts, but the results were not significant $(p=0.8$,

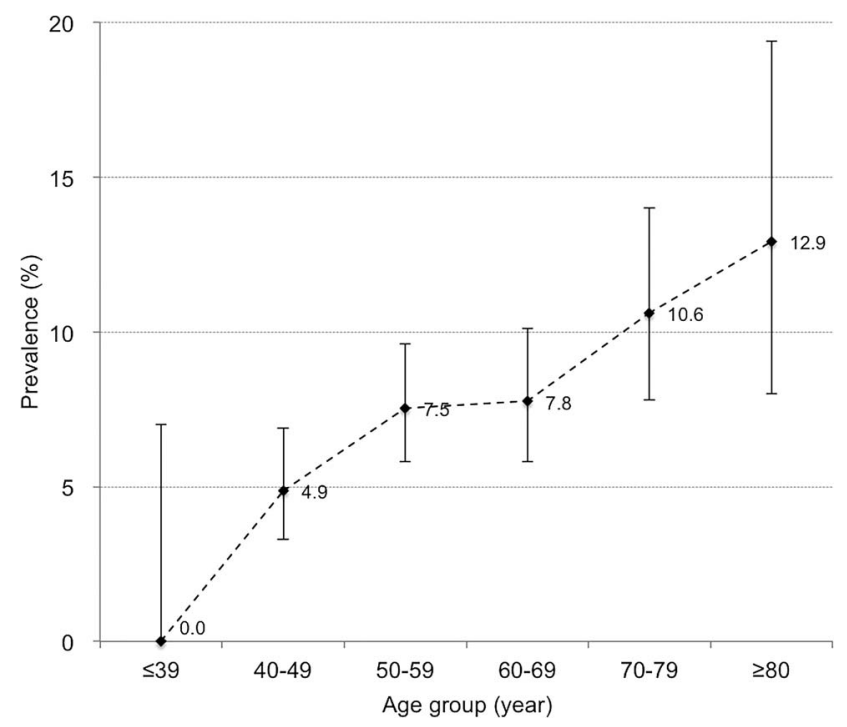

Figure 3 Prevalence of pulmonary cysts in association with age. Plotted dots indicate the prevalence in each age group. Vertical lines extending from each plot represent $95 \% \mathrm{Cl}$ with horizontal lines at the top and bottom representing upper and lower limits, respectively.
0.051). Participants with pulmonary cysts were further stratified with smoking status (table 3 ). There were no significant difference between non-smokers and smokers in demographic features (age, sex and BMI), coronary artery calcium score, cyst diameter and its interval changes $(p=0.2-0.98)$. Emphysema was more frequently seen in smokers $(\mathrm{p}=0.002)$.

Emphysema was detected in $13.3 \%$ (350/2633). The details of the associations between emphysema and participant demographics will be discussed in a separate study (unpublished data). Pulmonary cysts were observed in 7.1\% (25/350) of participants with emphysema (figure 2) and in 7.7\% (175/2283) of those without emphysema. There was no significant association between pulmonary cysts and emphysema $(p=0.7)$.

The prevalence of pulmonary cysts in different age decades is shown in figure 3 . The prevalence gradually increased with age, from $4.9 \%$ at age $40-49$ years to $12.9 \%$ at age over 80 years. No participants younger than 40 years showed pulmonary cysts.

Results of pulmonary function tests are summarised in table 4. Out of 2633 participants, 2468 had pulmonary function data (191 with pulmonary cysts and 2277 without). There was no significant difference in pulmonary functions $(p=0.07-0.6)$, except for DLCO $(p=0.03)$. Mean DLCO in those with pulmonary cysts was slightly but significantly reduced, although still within normal limits (94\% of predicted value).

Of 200 participants with pulmonary cysts on MDCT2, 80 did not have comparable CT scans of MDCT1, 109 pulmonary cysts were also identified with MDCT1, and 11 did not have any identifiable pulmonary cysts on MDCT1 (regarded as newly appeared on MDCT2). CCCs for intraobserver and interobserver agreement of measurements were substantial $(\mathrm{CCC}=0.99$ (95\% CI 0.98 to 0.99 ) and 0.99 (95\% CI 0.98 to 0.99 ), respectively). Bland-Altman plots showed that means of absolute difference of measurements were -0.07 (95\% CI -1.73 to 1.59 ) and -0.23 (95\% CI -1.68 to 1.22$)$ for intraobserver and interobserver variability (see online supplementary figures S1 and S2). These results validate the threshold criteria of interval change of $2 \mathrm{~mm}$. Interval changes of pulmonary cysts are shown in figure 4. Over the median interval period of 6.1 years, $57 \%$ $(68 / 120)$ showed no change, 33\% (39/120) increased in diameter (figure 5) and 9\% (11/120) were newly found in the latest 
Table 4 Results of pulmonary function test of the participants based on the presence of pulmonary cysts.

\begin{tabular}{|c|c|c|c|}
\hline & No pulmonary cysts $(\mathrm{N}=2277$ ) & Pulmonary cysts $(\mathrm{N}=191)$ & $p$ value ${ }^{*} \dagger$ \\
\hline $\mathrm{FEV}_{1}, \%$ of predicted value $\ddagger$ & $0.98 \pm 0.15$ & $0.99 \pm 0.15$ & 0.2 \\
\hline FVC, $\%$ of predicted value $\ddagger$ & $1.02 \pm 0.13$ & $1.03 \pm 0.13$ & 0.4 \\
\hline $\mathrm{FEV}_{1} / \mathrm{FVC}, \%$ of predicted value $\ddagger$ & $0.96 \pm 0.09$ & $0.96 \pm 0.09$ & 0.6 \\
\hline DLCO, \% of predicted value§ & $0.97 \pm 0.16(\mathrm{~N}=1895)$ & $0.94 \pm 0.15(\mathrm{~N}=167)$ & 0.03 \\
\hline TLCף, $\%$ of predicted value** & $0.84 \pm 0.15(\mathrm{~N}=2244)$ & $0.83 \pm 0.15(\mathrm{~N}=184)$ & 0.07 \\
\hline \multicolumn{4}{|c|}{ 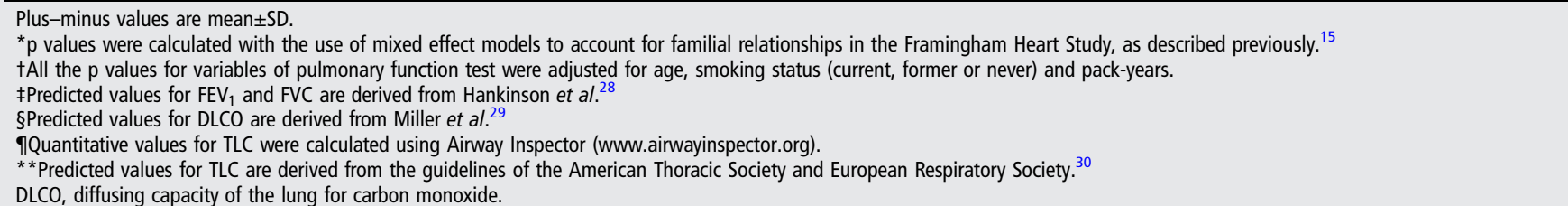 } \\
\hline
\end{tabular}

scans of MDCT2. Median diameter of pulmonary cysts on MDCT2 $(9.5 \mathrm{~mm})$ was slightly but significantly larger than that on MDCT1 (7.9 mm; p<0.001).

\section{DISCUSSION}

The present study reveals that the prevalence of pulmonary cysts in the FHS is $7.6 \%$, which increases with age. Pulmonary cysts appear in participants aged 40 years or older, but not in those younger than 40 years. Pulmonary cysts are most likely to appear solitary in the peripheral area of the lower lobes and mostly remain unchanged but may slightly increase in size over time. Pulmonary cysts do not result in changes in spirometry and are not associated with cigarette smoking and emphysema.

In the studies by Copley et $a l^{3}$ and Winter et $a l^{4}$ with relatively small numbers of subjects, only the elderly groups (older than 65 or 75 years) had pulmonary cysts. The present study of a large population-based cohort revealed that pulmonary cysts can be seen even in middle-aged participants as young as 40 years. The prevalence of pulmonary cysts is $7.6 \%$ in the FHS cohort (mean age 59.2 years), which increases with age (from $4.9 \%$ at $40-49$ to $12.9 \%$ at 80 or older). In addition, longitudinal observation revealed that most of the pulmonary cysts remain unchanged, but some may increase slightly in size. Pulmonary cysts did not result in reduced measures of spirometry. These findings may support the premise that pulmonary cysts found in asymptomatic individuals who are middle aged or older are likely to be part of the aging changes of the lungs.

Pulmonary cysts may somewhat arbitrarily refer to various conditions with parenchymal destruction depending on the context, including bullous emphysema. However, pulmonary cysts should be differentiated from emphysema because emphysema is one of the major aspects of COPD and its clinical
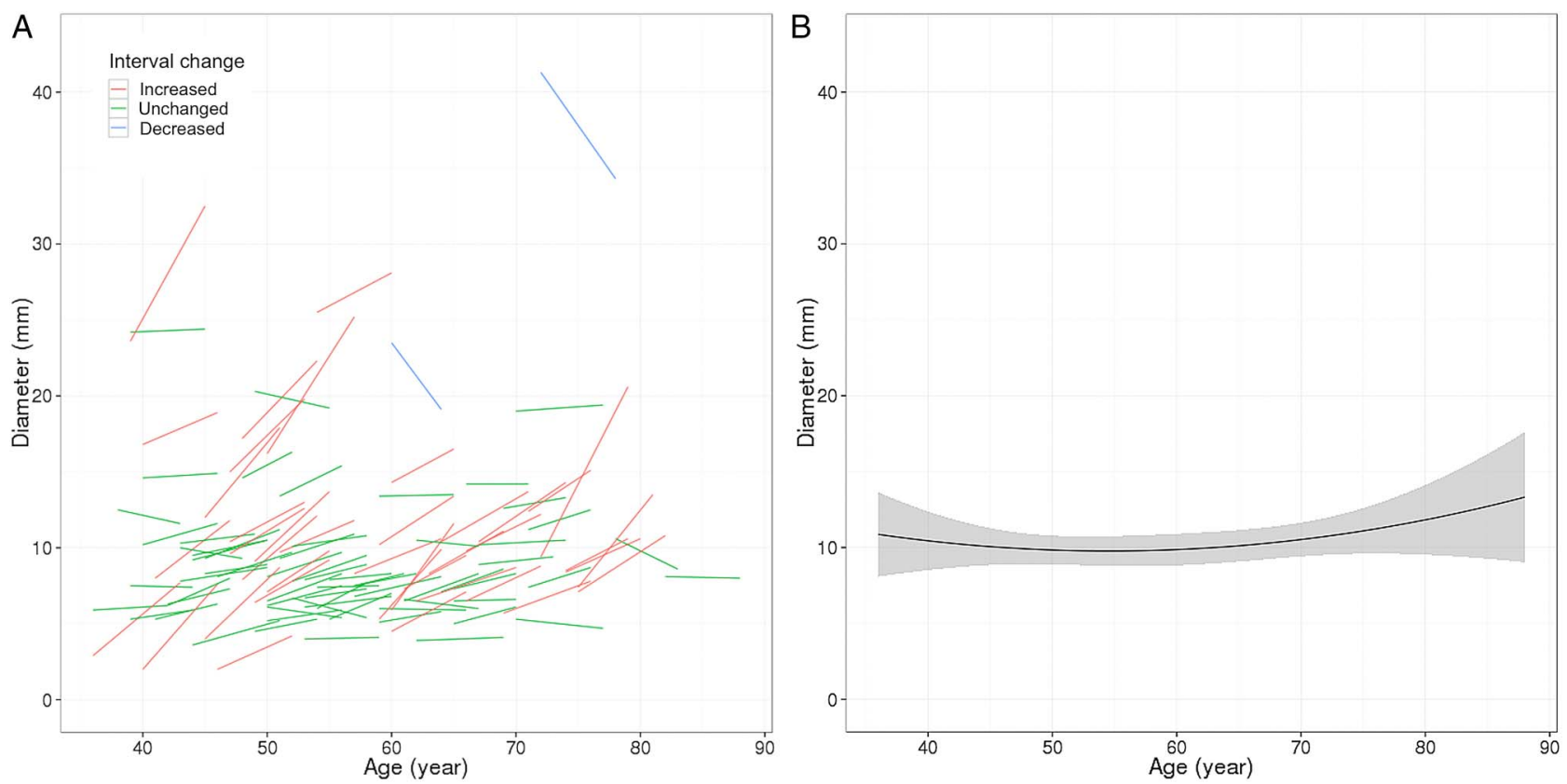

Figure 4 Longitudinal observations of pulmonary cysts. (A) Participants ( $\mathrm{N}=109)$ who had pulmonary cysts detected on both CT scans (MDCT1 and MDCT2, median interval of 6.1 years) are plotted for diameter of pulmonary cysts and participant's age. Each short line represents an interval change of a pulmonary cyst in diameter in each participant. Red lines $(\mathrm{N}=39)$ indicate increased in diameter, green lines $(\mathrm{N}=68)$ unchanged, and blue lines decreased $(\mathrm{N}=2)$. Increase or decrease in diameter was defined as changes in diameter of more than 2 mm accounting for measurement variability. (B) A curved-fit line represents an overall tendency, slight increase of the diameter of pulmonary cysts with age. Shaded area represents 95\% Cl. 


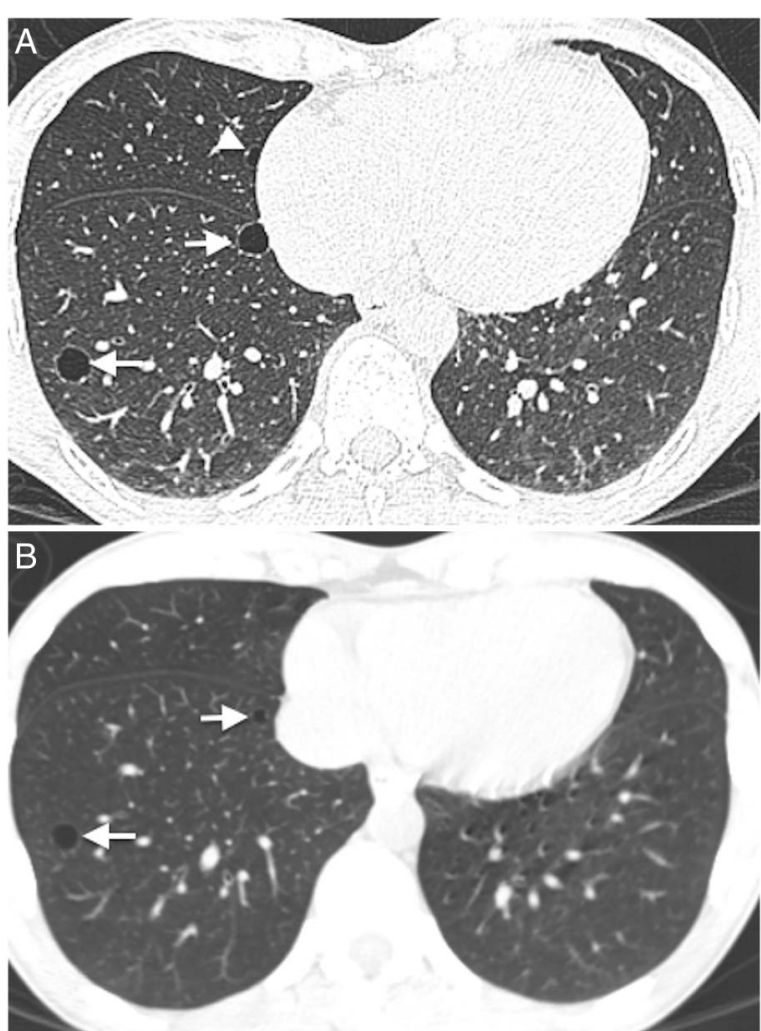

Figure 5 Transaxial CT images at the lower level (A) of a 51-year-old woman who has no smoking history show multiple thin-walled pulmonary cysts. These cysts are located predominantly in lower lobes, mostly in subpleural or peripheral areas. Comparison to the previous scan (B) (obtained 7.1 years previously) demonstrates an increase in size and number of cysts. Cysts in the right lower lobe ( $A$ and $B$, arrows) increased in size (medial: 5 to $10 \mathrm{~mm}$, lateral: 9 to $11 \mathrm{~mm}$ ). The cyst in the middle lobe ( $A$, arrowhead) was not detected on the previous image (B).

impact is of great significance. ${ }^{16}$ Identification of a thin wall on CT may differentiate pulmonary cysts from emphysema. In addition, our study involved a direct comparison of the groups with emphysema and pulmonary cysts separately diagnosed on CT and revealed that these two entities occur independently. In the previous studies, it was not possible to discuss the association between age-related pulmonary cysts and emphysema because the presence of chronic lung diseases including emphysema was a part of exclusion criteria. ${ }^{3} 4617$ Furthermore, some other findings also bolster the fact that pulmonary cysts are a distinct entity from emphysema: pulmonary cysts are not associated with smoking and tend to occur with the lower lobe predilection with no sex difference and no impairment of measures of spirometry, whereas emphysema predominantly occurs in the upper lobes, in male smokers. ${ }^{18}$ However, there might be possible overlaps on CT findings between these two entities because compressed lung parenchyma adjacent to emphysematous lesions may show increased density, mimicking the thin wall of pulmonary cysts, which probably represent atelectasis or interlobular septa. ${ }^{19}$ That is one of our motives for investigating vascular penetration: we expected that the finding could be suggestive of centrilobular emphysema because centrilobular pulmonary arteries or arterioles indicate the centre of each lobule. $^{19}{ }^{20}$ However, there was no significant association detected between the specific image finding and the presence of emphysema.
Although pulmonary cysts are most likely to be solitary, they could be sporadic or sometimes multiple. The prevalence of multiple pulmonary cysts was $0.9 \%$ of all participants. In such cases, it is important to clinically exclude the possibility of the cystic lung diseases, such as PLCH, LAM and LIP. Age is obviously useful for the differentiation. PLCH typically affects young adult smokers ${ }^{21}$ and LAM primarily affects women of childbearing age, ${ }^{22} 23$ whereas pulmonary cysts associated with aging rarely occur in these age groups. PLCH may appear with irregular cysts with a thicker wall in the upper lobes with or without nodular lesions. LAM may appear with a myriad of small cysts, ${ }^{22}$ whereas pulmonary cysts associated with aging mostly occur solitarily or in small numbers. LIP occurs in adults of middle age. ${ }^{22} 24$ However, associated image findings to LIP, such as diffuse or multifocal ground-grass opacities, thickening of bronchovascular bundles, and interlobular septal thickening, help differentiate it from age-related pulmonary cysts.

Notably, participants with pulmonary cysts showed significantly lower BMI than those without pulmonary cysts. A low $\mathrm{BMI}$ is known to be associated with the presence of emphysema, and nutritional depletion is regarded as one of the possible causes of emphysema. ${ }^{25}$ Coxson et $a l^{26}$ revealed that decreases in BMI and DLCO are correlated with emphysema demonstrated with qualitative CT analysis. Therefore, nutritional status influences the destruction of lung parenchyma and may play some roles in the development of pulmonary cysts as we found lower BMI in participants with pulmonary cysts. Our results showed that mean DLCO in those with pulmonary cysts was slightly but significantly lower, although within normal limits. We revealed pulmonary cysts are not associated with emphysema, and it is unlikely that small numbers of cysts may affect general lung function; in fact, $\mathrm{FEV}_{1}$ and FVC were not impaired. Therefore, the slight decrease in DLCO should be explained by something other than lung parenchymal destruction, for example, pulmonary vascular disease or anaemic status. Our data do not strongly suggest that measures of coronary artery disease (eg, coronary artery calcium scores) influence the development of pulmonary cysts as coronary artery calcium scores were rather reduced among those with pulmonary cysts. Another possible cause would be interstitial lung abnormalities (ILA) accompanied by pulmonary cysts. ${ }^{11}$ However, additional analysis of ILA and pulmonary cysts in the present cohort did not reveal any significant association. Further study is necessary to induce rational explanations for the cause of pulmonary cysts and the association between DLCO change and pulmonary cysts.

There are several limitations in the present study. First, our study lacks histopathological confirmation and mostly relies on CT findings. Therefore, the possibility that our cohort incidentally included participants with primary cystic lung diseases cannot be totally ruled out, although most of the participants were supposedly healthy individuals. Second, comparison of CT scans is not complete: not all of the cases with pulmonary cysts had comparable CT scans, which are cardiac scans with different protocols and insufficient coverage of the lungs. However, this is the first study that involved the largest number of participants with longitudinal observations of pulmonary cysts. Lastly, the FHS cohorts could be biased because participants of the FHS could be healthier than the general population due to substantial medical support and high health consciousness. However, investigation of the FHS cohorts provides a unique opportunity of epidemiological significance to investigate normal or aging changes such as pulmonary cysts, and makes this study distinctive from previous investigations of volunteers or patients recruited at hospital. 
In conclusion, pulmonary cysts were identified in $7.6 \%$ on chest CT in the FHS and were associated with increased age. They were distinct from emphysema and not associated with smoking or impairment in measures of spirometry, suggesting that these pulmonary cysts could be mostly explained by the normal aging process of the lungs. The explanation of the associated decreases in DLCO and BMI requires further investigation. Multiple pulmonary cysts with its count of five or more may need to be scrutinised for the possibility of cystic lung diseases.

Acknowledgements The authors acknowledge Alba Cid, MS for editorial work on the manuscript.

Contributors All the authors of this manuscript fulfil the following four criteria: substantial contributions to the conception or design of the work, or the acquisition, analysis, or interpretation of data for the work; drafting the work or revising it critically for important intellectual content; final approval of the version to be published; agreement to be accountable for all aspects of the work in ensuring that questions related to the accuracy or integrity of any part of the work are appropriately investigated and resolved.

Funding MN is supported by NCl Grant Number: 1K23CA157631. GRW is supported by NIH Grant Number: R01 HL116473, R01 HL107246 and P01 HL114501. GMH is supported by NIH Grant Number: K08 HL092222, U01 HL105371, P01 HL114501 and R01 HL111024. HH is supported by NIH Grant Number: K25 HL104085 and R01 HL116473. This work was partially supported by the NHLBI's Framingham Heart Study contract N01-HC-25195 and R01 HL111024.

Competing interests None declared.

Patient consent Obtained.

Ethics approval Brigham and Women's Hospital, Boston University.

Provenance and peer review Not commissioned; externally peer reviewed.

Data sharing statement We included unpublished data for emphysema derived from the same cohorts we involved in the present study. One of the authors is working on analysis of the data and preparation for publication. All the unpublished data are available from the corresponding author of the study.

\section{REFERENCES}

1 Hansell DM, Bankier AA, MacMahon $\mathrm{H}$, et al. Fleischner Society: glossary of terms for thoracic imaging. Radiology 2008;246:697-722.

2 Elicker BM, Webb WR. Fundamentals of high-resolution lung CT: common findings, common patterns, common diseases and differential diagnosis. Lippincott Williams \& Wilkins, 2013

3 Copley SJ, Wells AU, Hawtin KE, et al. Lung morphology in the elderly: comparative CT study of subjects over 75 years old versus those under 55 years old. Radiology 2009;251:566-73.

4 Winter DH, Manzini M, Salge JM, et al. Aging of the lungs in asymptomatic lifelong nonsmokers: findings on HRCT. Lung 2015;193:283-90.

5 Vehmas T, Kivisaari L, Huuskonen MS, et al. Scoring CT/HRCT findings among asbestos-exposed workers: effects of patient's age, body mass index and common laboratory test results. Eur Radiol 2005;15:213-19.

6 Copley SJ, Giannarou S, Schmid VJ, et al. Effect of aging on lung structure in vivo: assessment with densitometric and fractal analysis of high-resolution computed tomography data. J Thorac Imaging 2012;27:366-71.
7 Matsuoka S, Uchiyama K, Shima $\mathrm{H}$, et al. Bronchoarterial ratio and bronchial wall thickness on high-resolution $C T$ in asymptomatic subjects: correlation with age and smoking. AJR Am J Roentgenol 2003;180:513-18.

8 Lee KW, Chung SY, Yang I, et al. Correlation of aging and smoking with air trapping at thin-section CT of the lung in asymptomatic subjects. Radiology 2000;214:831-6.

9 Mendis S. The contribution of the Framingham Heart Study to the prevention of cardiovascular disease: a global perspective. Prog Cardiovasc Dis 2010;53:10-14.

10 Washko GR, Lynch DA, Matsuoka S, et al. Identification of early interstitial lung disease in smokers from the COPDGene Study. Acad Radiol 2010;17:48-53.

11 Hunninghake GM, Hatabu H, Okajima Y, et al. MUC5B promoter polymorphism and interstitial lung abnormalities. N Engl J Med 2013;368:2192-200.

12 Revel M-P, Bissery A, Bienvenu $M$, et al. Are two-dimensional CT measurements of small noncalcified pulmonary nodules reliable? Radiology 2004;231:453-8.

13 Kundel HL, Polansky M. Measurement of observer agreement. Radiology 2003;228:303-8.

14 Araki T, Sholl LM, Gerbaudo VH, et al. Thymic measurements in pathologically proven normal thymus and thymic hyperplasia: intraobserver and interobserver variabilities. Acad Radiol 2014;21:733-42.

15 Uh HW, Wijk HJ, Houwing-Duistermaat JJ. Testing for genetic association taking into account phenotypic information of relatives. BMC Proc 2009:3(Suppl 7):S123.

16 Ford ES, Croft JB, Mannino DM, et al. COPD surveillance-United States, 19992011. Chest 2013;144:284-305.

17 Well DS, Meier JM, Mahne A, et al. Detection of age-related changes in thoracic structure and function by computed tomography, magnetic resonance imaging, and positron emission tomography. Semin Nucl Med 2007;37:103-19.

18 Araki T, Nishino M, Zazueta OE, et al. Paraseptal emphysema: prevalence and distribution on CT and association with interstitial lung abnormalities. Eur J Radiol 2015;84:1413-18.

19 Lynch DA, Austin JHM, Hogg JC, et al. CT-definable subtypes of chronic obstructive pulmonary disease: a statement of the Fleischner Society. Radiology 2015:277:192-205.

20 Murata K, Itoh H, Todo G, et al. Centrilobular lesions of the lung: demonstration by high-resolution CT and pathologic correlation. Radiology 1986;161:641-5.

21 Abbott GF, Rosado-de-Christenson ML, Franks TJ, et al. From the archives of the AFIP: pulmonary Langerhans cell histiocytosis. Radiographics 2004;24:821-41.

22 Cantin L, Bankier AA, Eisenberg RL. Multiple cystlike lung lesions in the adult. AJR Am J Roentgenol 2010;194:W1-11.

23 Ryu JH, Moss J, Beck GJ, et al. The NHLBI lymphangioleiomyomatosis registry: characteristics of 230 patients at enrollment. Am J Respir Crit Care Med 2006;173:105-11.

24 Johkoh T, Müller NL, Pickford HA, et al. Lymphocytic interstitial pneumonia: thin-section CT findings in 22 patients. Radiology 1999;212:567-72.

25 Ogawa E, Nakano $\mathrm{Y}$, Ohara $\mathrm{T}$, et al. Body mass index in male patients with COPD: correlation with low attenuation areas on CT. Thorax 2009;64:20-5.

26 Coxson $\mathrm{HO}$, Chan $\mathrm{IH}$, Mayo JR, et al. Early emphysema in patients with anorexia nervosa. Am J Respir Crit Care Med 2004;170:748-52.

27 Agatston AS, Janowitz WR, Hildner FJ, et al. Quantification of coronary artery calcium using ultrafast computed tomography. J Am Coll Cardiol 1990;15:827-32.

28 Hankinson $J \mathrm{~L}$, Odencrantz JR, Fedan KB. Spirometric reference values from a sample of the general U.S. population. Am J Respir Crit Care Med 1999;159:179-87.

29 Miller A, Thornton JC, Warshaw R, et al. Single breath diffusing capacity in a representative sample of the population of Michigan, a large industrial state. Predicted values, lower limits of normal, and frequencies of abnormality by smoking history. Am J Respir Dis 1983;127:270-7.

30 Stocks J, Quanjer PH. Reference values for residual volume, functional residual capacity and total lung capacity. ATS Workshop on Lung Volume Measurements. Official Statement of The European Respiratory Society. Eur Respir J 1995;8:492-506. 